\title{
Design, performance, and potential scientific applications of the evanescent wave coronagraph with an adjustable inner working angle
}

Mary Angelie Alagao, Christophe Buisset, Adithep Kawinkij, Apirat Prasit, Thierry Lepine, et al.

Mary Angelie Alagao, Christophe Buisset, Adithep Kawinkij, Apirat Prasit, Thierry Lepine, Yves Rabbia, Anthony Berdeu, Eric Thiebaut, Maud Langlois, Michel Tallon, Supachai Awiphan, Eugene Semenko, Pakakaew Rittipruk, David Mkrtichian, Saran Poshyachinda, Boonrucksar Soonthornthum, "Design, performance, and potential scientific applications of the evanescent wave coronagraph with an adjustable inner working angle," Proc. SPIE 11852, International Conference on Space Optics - ICSO 2020, 118523W (11 June 2021); doi: 10.1117/12.2599626

SPIE Event: International Conference on Space Optics - ICSO 2021, 2021, Online Only 


\title{
International Conference on Space Optics-ICSO 2020
}

Virtual Conference

30 March-2 April 2021

Edited by Bruno Cugny, Zoran Sodnik, and Nikos Karafolas
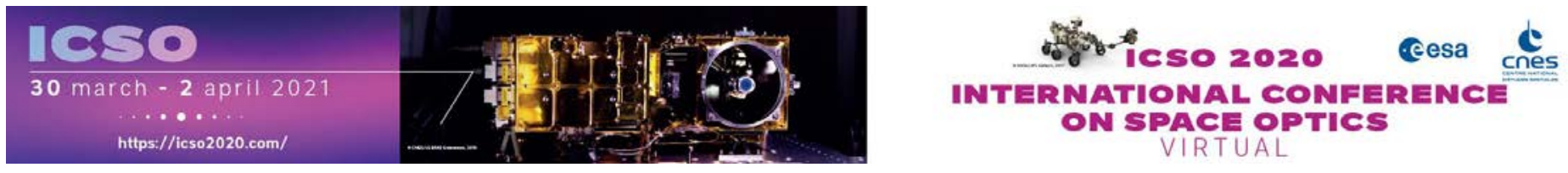

\begin{abstract}
Design, performance, and potential scientific applications of the evanescent wave coronagraph with an adjustable inner working angle
\end{abstract}

\section{Cesa iss poecestings lenes}




\title{
Design, performance and potential scientific applications of the Evanescent Wave Coronagraph with an adjustable Inner Working Angle
}

Mary Angelie Alagao ${ }^{\mathrm{a}, \mathrm{b}, \mathrm{c}, \mathrm{g}}$, Christophe Buisset ${ }^{\mathrm{a}}$, Adithep Kawinkij ${ }^{\mathrm{a}}$, Apirat Prasit ${ }^{\mathrm{a}}$, Thierry Lepine $^{\mathrm{b}, \mathrm{c}}$, Yves Rabbia ${ }^{\mathrm{d}}$, Anthony Berdeu ${ }^{\mathrm{a}, \mathrm{e}}$, Eric Thiebaut ${ }^{\mathrm{f}}$, Maud Langlois ${ }^{\mathrm{f}}$, Michel Tallon ${ }^{\mathrm{f}}$, Supachai Awiphan ${ }^{\mathrm{a}}$, Eugene Semenko ${ }^{\mathrm{a}}$, Pakakaew Rittipruk ${ }^{\mathrm{a}}$, David Mkrtichian ${ }^{\mathrm{a}}$, Saran Poshyachinda $^{\mathrm{a}}$, and Boonrucksar Soonthornthum ${ }^{\mathrm{a}}$

${ }^{a}$ National Astronomical Research Institute of Thailand , 260 Moo 4, T. Donkaew, A. Maerim , Chiang Mai, Thailand, 50180

b'Institut d'Optique Graduate School , 18 Rue Benoit Lauras, Saint-Etienne, France 42000 ${ }^{c}$ Hubert Curien Laboratory (CNRS, UMR 5516) 18 Rue Benoit Lauras, Saint-Etienne, France 42000

dUniversité de la Côte d' Azur, OCA, CNRS, Lagrange, Boulevard de l'Observatoire, B.P. 4229 F-06304 NICE Cedex-43, France

eDepartment of Physics, Faculty of Science, Chulalongkorn University, 254 Phayathai Road, Pathumwan, Bangkok, Thailand 10330

${ }^{\mathrm{f}}$ Univ Lyon, Univ Lyon1, Ens de Lyon, CNRS, Centre de Recherche Astrophysique de Lyon UMR5574, F-69230, Saint-Genis-Laval, France

${ }^{g}$ PhD Program in Astronomy, Department of Physics and Materials Science, Faculty of Science, Chiang Mai University, Chiang Mai, Thailand, 50200

\begin{abstract}
The Center for Optics and Photonics of the National Astronomical Research Institute of Thailand, together with the Institut d'Optique Graduate School and the Centre de Recherche Astrohpysique de Lyon (CRAL), is currently developing the Evanescent Wave Coronagraph (EvWaCo). The coronagraph relies on the tunneling effect to produce a fully achromatic focal plane mask (FPM) with an adjustable size. The full instrument comprises a coronagraph and adaptive optics system that will be mounted on the Thai National Telescope and is specified to reach a raw contrast of $10^{-4}$ at an inner working angle of 3 Airy radii. The coronagraph will be used to perform high contrast observations of stellar systems during on-sky observations over the spectral domain $[600 \mathrm{~nm}, 900 \mathrm{~nm}]$. In this paper, we present the opto-mechanical design of the EvWaCo prototype and the performance measured in laboratory conditions. We also discuss the potential applications for space-based observations and the development plan under this project in the next five years.
\end{abstract}

Keywords: coronagraph, high contrast imaging, adaptive optics

\section{INTRODUCTION}

Direct imaging provides a toolkit in characterizing the close environment of stars. In particular, it allows us to study the orbital configuration and the atmospheric chemical composition of exoplanets. However, the small angular separation and the high contrast between exoplanets and their host stars pose several technological constraints. ${ }^{1}$

To alleviate these constraints, coronagraphs, equipped with an adaptive optics system, are used to suppress the light from host stars. Current state-of-the-art ground-based instruments such as the Gemini Planet Imager

Further author information: (Send correspondence to A.A.A.)

A.A.A.: E-mail: angelie@narit.or.th, Telephone: 053121268 
(GPI) and the Spectro-Polarimetric High Contrast Exoplanet Research (SPHERE) have already achieved better than $10^{-4}$ normalized contrast as close as 0.4 " in the near-infrared wavelengths. ${ }^{2,3}$ With this performance, gas giant planets and brown dwarfs orbiting nearby young stars can already be detected. ${ }^{4,5}$

One of the projects of the NARIT Center for Optics and Photonics and the Thai Space Consortium is the Evanescent Wave Coronagraph (EvWaCo) project. The goal of this project is to develop an EvWaCo prototype that will be installed at the Thai National Telescope. This coronagraph relies on the principle of frustrated total internal reflection to suppress the star light. ${ }^{6-8}$ It will observe through an unobstructed elliptical aperture with a major axis equal to $1.2 \mathrm{~m}$ and a minor axis equal to $0.8 \mathrm{~m}$. The focal plane mask is composed of a lens and a prism placed in contact with each other. Depending on the pressure between the lens and the prism, the mask size can be adjusted that allows the fine-tuning of the inner working angle (IWA). Moreoever, the mask inherently adapts itself to the wavelength, thus, providing an achromatic rejection. ${ }^{9-11}$

In this paper, we present the current status of this project. In particular, we describe the prototype that is currently in development and the performance of the coronagraph in the laboratory. In addition, we provide a development plan to reach state-of-the-art performance suitable for space applications.

\section{THE EVWACO PROTOTYPE}

The EvWaCo prototype is designed to work on an unobscured sub-aperture of the $2.4 \mathrm{~m}$ Thai National Telescope (TNT). This prototype will be vertically installed in one of the ports of the Instrument Cube (IC) of the Thai National Telescope. Figure 1 illustrates the installed prototype on the TNT and the contents of the prototype. The prototype can be classified into two major sub-systems: optical and mechanical. The EvWaCo prototype is a coronagraph equipped with an adaptive optics system.
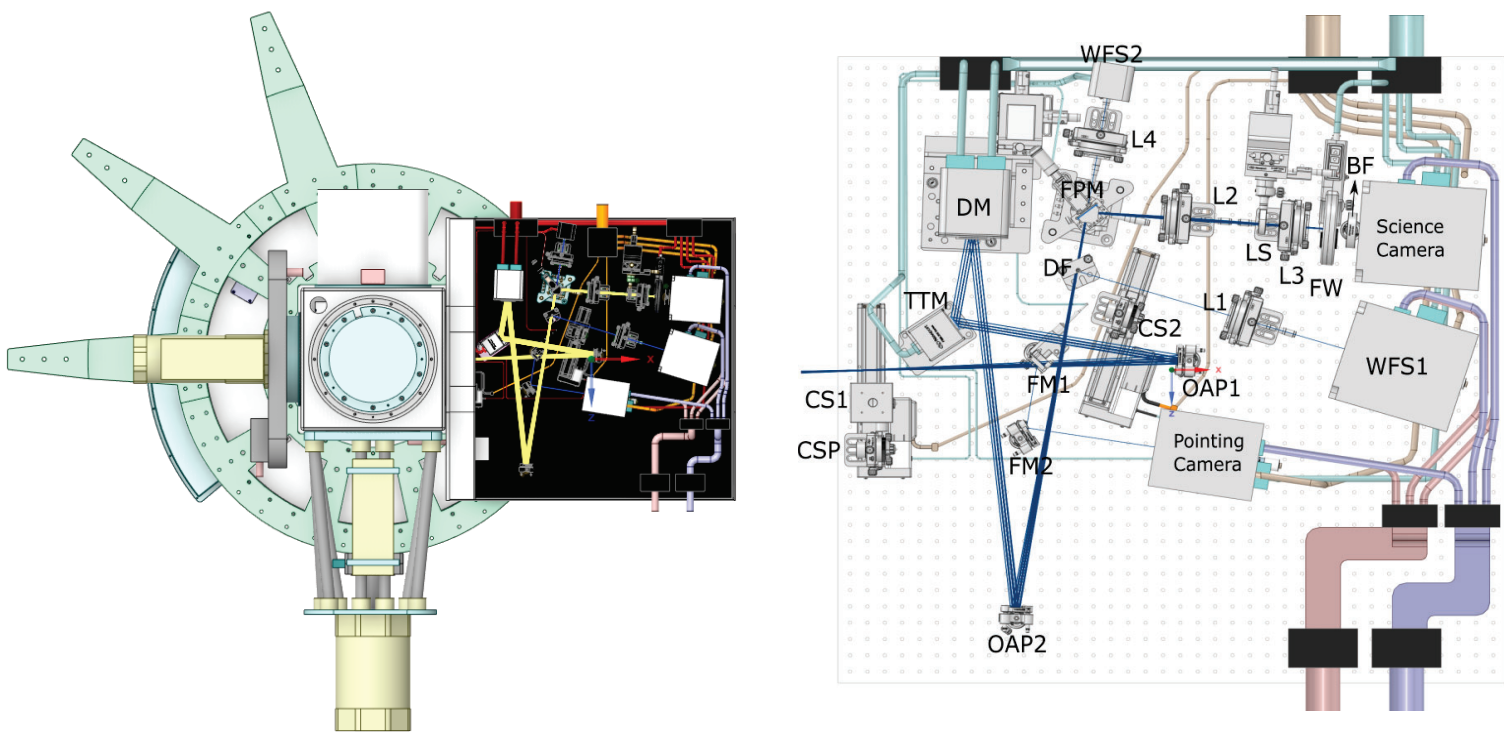

Figure 1. (left) EvWaCo Prototype on the Thai National Telescope installed vertically and (right) inside the EvWaCo prototype. The following are the components inside the prototype: calibration source 1 (CS1), calibrating sphere (CSP), off-axis parabola 1 (OAP1), off-axis parabola 2 (OAP2), flip mirror 1 (FM1), folding mirror 2 (FM2), tip-tilt mirror (TTM), deformable mirror (DM), dichroic filter (DF), calibration source 2 (CS2), collimating lens 1 (L1), wavefront sensor 1 (WFS1), focal plane mask (FPM), collimating lens 2 (L2), Lyot stop (LS), focusing lens 3 (L3), filter wheel (FW), broadband filter (BF), science camera, collimating lens 4 (L4), and wavefront sensor 2 (WFS2).

\subsection{Mechanical Structure}

The mechanical structure of the EvWaCo prototype takes into account the constraints imposed by the optical tolerancing and the limitations of the IC. The main mechanical requirements of the prototype are the following: 
i) a maximum deformation of the breadboard interfaces for each opto-mechanical component should be less than 150 microns over all orientations of the IC and a temperature variation of $25^{\circ} \mathrm{C}$, ii) a gross weight of less than $180.0 \mathrm{~kg}$, and iii) a maximum volume of $1000 \mathrm{~mm} \times 1000 \mathrm{~mm} \times 450 \mathrm{~mm}$.

The opto-mechanical components are customized individually based on their given requirements and specifications. Using load analysis, the weight of each opto-mechanical component is estimated based on their individual macrostructures. This load information serves as the basis for the design of the prototype mechanical structure and is used in distributing the weight budget of the design.

To comply with the requirements, we selected and procured a full-body carbon fiber reinforced polymer (CFRP) breadboard from CarbonVision GmbH due to its superior specific modulus and thermal stability compared to typical aluminum alloy breadboards in the market. The volumes of the opto-mechanical components and the breadboard drive the dimension of prototype enclosure. Some sections of the volume outline are topologically removed to allow a significant gain by trading off the improvement of overall deformation with the gross weight of the prototype. The structure of the prototype is constructed from space-grade and aviation-grade materials with high specific modulus and low thermal expansion such as 6AI-4V titanium alloy (Grade 5), commercially pure titanium alloy (Grade 1 or 2) and CFRP. The mechanical parts include a set of plates, angles, screws, nuts and bolts for machinability and feasibility.

To minimize the deformation due to the gravity, a honeycomb sandwich configuration is introduced in the critical structure to reduce the dominant deflection caused by the compressive stress. The sandwich is feasibly designed as an extruded structure which can be fabricated using a Computer Numerical Control (CNC) machine.

Additionally, to compensate the deformation due to the ambient thermal variation, thermal compensation nodes are used to form an athermal configuration. The technique utilizes the interfacial structures between the breadboard and the main structure that comprises 9 compensation nodes. These nodes are constructed such that their unique configuration not only connect the main components, but also compensate the thermal deformation of the structure. Moreoever, they are designed to be changeable and finely tunable in terms of the thermal compensation magnitude.

Besides the structure, the prototype also requires additional supporting sub-systems that are essential to sustain and communicate with the EvWaCo including: the power supply, cooling circulation and information channel. The power supply routes the electricity toward the electronic components in the prototype through an allocated power inlet. The cooling circulation directs the hot water from the cameras inside the prototype to an external refrigeration device. This minimizes the interior local air turbulence. Lastly, the information channel provides an interface to exchange information between the EvWaCo and external communication devices. More details regarding the mechanical structure of the prototype will be described in a future paper.

\subsection{Optical Design}

Table 1 lists the optical specifications of the EvWaCo prototype. The propagation of light inside the prototype is described as follows. The light beam provided by the telescope is located $65 \mathrm{~mm}$ away from the Instrument Cube flange. We plan to calibrate the prototype by placing a calibration source CS1 at TNT focus. CS1 consists of an LED connected to a single mode fiber and is installed in a translation rail together with the calibration sphere CSP. The first off-axis parabola OAP1 reflects the beam towards the tip-tilt mirror TTM and then to the deformable DM. The DM that constitutes the aperture stop of the coronagraph reflects the beam towards a second off-axis parabola OAP2. From the OAP2, a dichroic filter DC reflects the short wavelengths lower than $600 \mathrm{~nm}$ towards the first wavefront sensor WFS1 and transmits the longer wavelengths towards the FPM. The reflected beam goes toward a collimating lens L1 and then to the first wavefront sensor (WFS1). The beam transmitted is focused at the hypotenuse of the prism in the focal plane mask FPM. At this plane, the beam reflected by the FPM goes towards the collimating lens L2, then to the Lyot stop and, finally, focused by the lens L3 towards the science camera. A filter wheel and a broadband filter are placed in front of the camera. The filter wheel houses a neutral density filter and another lens to obtain a pupil image of the setup. Meanwhile, the beam transmitted by the FPM is collimated by a lens L4 towards a second wavefront sensor WFS2.

A pointing camera is also installed and will be used to point the TNT and ensure that the target is centered at the EvWaCo FOV. Another calibration source CS2 is installed in front of L1 for the calibration of the WFS1. 
Table 1. Optical Specifications of the EvWaCo Prototype.

\begin{tabular}{|l|l|l|}
\hline Parameter & Specification & Comments \\
\hline Science channel spectral band & R- and I- bands & Goal: V-, R- and I-bands \\
\hline Strehl ratio & SR $\geq 0.8$ & $\lambda=800 \mathrm{~nm}$, magnitude $m_{v}=8, \mathrm{~s} \approx 1 "$ \\
\hline AO loop frequency & $\geq 1 \mathrm{kHz}$ & Atmospherical effect real time compensation \\
\hline Entrance pupil shape and size & $1.2 \mathrm{~m} \mathrm{x} 0.8 \mathrm{~m}$ & Unobstructed pupil \\
\hline DM number of actuators & 192 actuators & 16 actuators across pupil major axis \\
\hline Raw contrast & $\leq 10^{-4}$ at IWA & $\Delta m \approx 10$, limited by WFE high frequencies \\
\hline Inner working angle & $0.5 "$ & Target: $\leq 0.3 "(2 \lambda / D$ at $800 \mathrm{~nm})$ \\
\hline FOV & $10^{\prime \prime}$ & AO active area and star halo \\
\hline Plate scale & $0.08^{\prime \prime}$ & 2 pixels per $\lambda / D$ \\
\hline Central object limiting magnitude & 8 & SR $\approx 0.8$ in I-band \\
\hline
\end{tabular}

\section{LABORATORY PERFORMANCE}

We have developed an EvWaCo testbed to measure the contrast performance obtained by the coronagraph using an upgraded focal plane mask. ${ }^{12}$ This testbed is on a passive setup and with no adaptive optics installed. In this paper, the raw contrast is calculated by dividing the normalized irradiance by the mask transmission where the normalized irradiance is the ratio between the star residual signal and the star PSF peak. ${ }^{13}$ Meanwhile, the radial distance is expressed in terms of $\lambda / D$ where $\lambda$ is the source wavelength and $\mathrm{D}$ is the diameter of the aperture stop.

The new focal plane mask consists of an upgraded mechanical structure that controls the pressure between the lens and the prism as shown in Figure 2 (left). Through this mechanism, the size of the mask and, therefore, the IWA can be adjusted. Figure 3 shows the 2D irradiance distribution of the mask transmission as the pressure between the lens and the prism is increased. The images were obtained using a source with a central wavelength of $780 \mathrm{~nm}$ and a spectral bandwidth of $3.2 \%$. On the same figure, the raw contrast performance for each mask is also calculated. We observe that the mask with the smallest IWA reaches the best contrast performance.
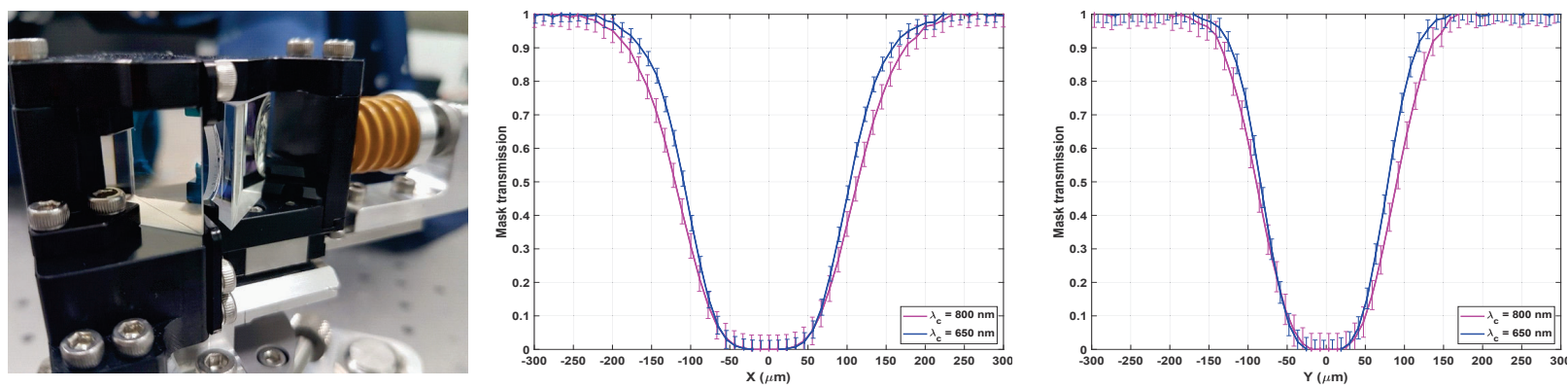

Figure 2. (Left) Focal plane mask installed in the testbed and the cross-section along the x-axis (middle) and the y-axis (right) of the mask transmission.

Figure 4 shows the normalized irradiance (top) and the corresponding raw contrast curves over the full I-band $\left(\lambda_{C} \approx 800 \mathrm{~nm}, \Delta \lambda / \lambda_{C} \approx 20 \%\right)$ and the full R-band $\left(\lambda_{C} \approx 650 \mathrm{~nm}, \Delta \lambda / \lambda_{C} \approx 23 \%\right)$. The corresponding mask transmission at the central wavelengths of these photometric bands are shown in Figure 2 (middle and right panel). We observe that the mask enlarges as the wavelength is increased from $650 \mathrm{~nm}$ to $800 \mathrm{~nm}$. In the I-band, the IWA is at $4.00 \lambda / D$ along the $\mathrm{x}$-axis and $3.00 \lambda / D$ along the $\mathrm{y}$-axis. Meanwhile, the IWA is at $4.27 \lambda / D$ along the $\mathrm{x}$-axis and $3.24 \lambda / D$ along the $\mathrm{y}$-axis in the $\mathrm{R}$-band. The results show that we are able to reach a raw 

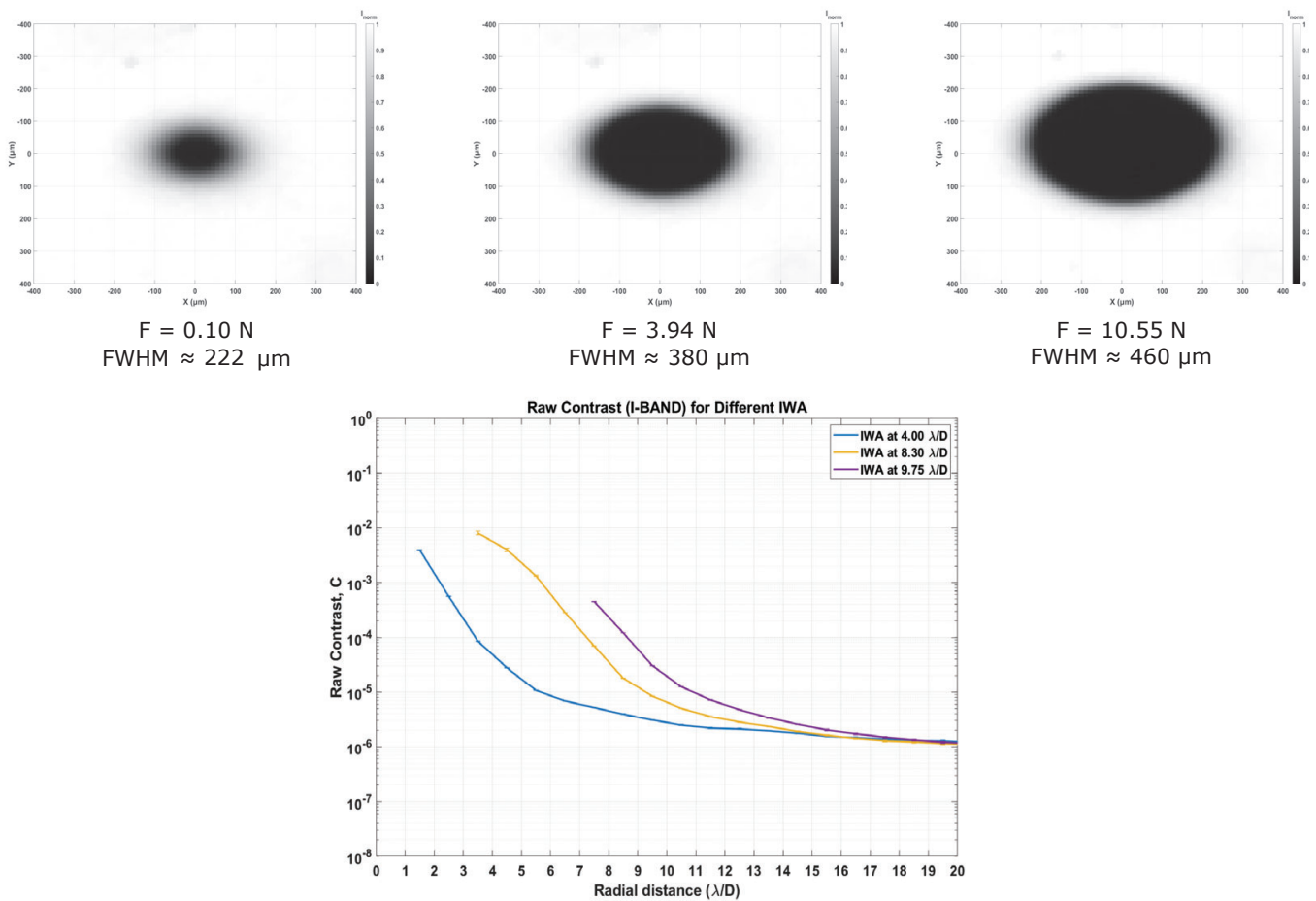

Figure 3. (Top) Mask transmission at increasing pressure applied by the lens on the prism.(Bottom) Raw Contrast Performance for different inner working angle

contrast close to $10^{-4}$ at $3 \lambda / D$ in the I-band and $4 \lambda / D$ in the R-band. More results on the characterization of this testbed can be found in Alagao et. al. $(2021) .{ }^{12}$

\section{DISCUSSION}

EvWaCo has three main advantages: i) the mask adapts inherently to the wavelength, ii) both the light from the star and the companion can be collected, and iii) the size of the mask can finely tuned to fit the size of the observed point-size or an extended object. Its quasi-achromatic capability provides a raw contrast performance, $\mathrm{C} \approx 10^{-4}$, as close as $3 \lambda / D$ in the I-band and $4 \lambda / D$ in the R-band.

The use of the coronagraph is not limited to the detection and characterization of exoplanets. The coronagraph can also be used to detect quasars and active galactic nuclei (AGNs), protoplanetary disks in young stars and debris disks in evolved star. ${ }^{14}$ Thus, the capability to fine-tune the IWA would be useful in the high contrast imaging of extended objects.

In space, the Space Telescope Imaging Spectrograph (STIS) installed at the Hubble Space Telescope coupled with focal plane wedges and a Lyot stop provides coronagraphy over a bandpass of $0.2 \mu \mathrm{m}-1.0 \mu \mathrm{m}$. The instrument is used to image nebulosity around stars in the range $0.34 \leq \mathrm{V} \leq 14$ and $-0.03 \leq \mathrm{B}-\mathrm{V} \leq 1.65 .{ }^{15}$ One of its occulters is the BAR5 that has an inner working angle of $0.2 "(3 \lambda / D$ at $\lambda=800 \mathrm{~nm})$. The median raw contrast performance obtained for $\delta$ Dor and HD 191849 varies between $\mathrm{C} \approx 10^{-3}$ at the IWA to $\mathrm{C} \approx 10^{-4}$ at $0.7^{\prime \prime}$ (10 $\lambda / \mathrm{D}) .{ }^{16}$ At these distances, the current EvWaCo testbed raw contrast performance on a passive setup showed a variation of $\mathrm{C} \approx 2 \cdot 10^{-4}$ to $\mathrm{C} \approx 3 \cdot 10^{-6}$ in the $\mathrm{I}$-band and $\mathrm{C} \approx 2 \cdot 10^{-3}$ to $\mathrm{C} \approx 10^{-5}$ in the $\mathrm{R}$-band.

Moreover, the Hubble Space Telescope does a small-angle maneuver to center the star at any position under any of the two opaque orthogonal wedges inside the 50Coron aperture. The wedges vary in width from $7.5 \lambda / D$ $(0.5 ")$ and $45 \lambda / D(3 ")$ at $\lambda \approx 800 \mathrm{~nm} \cdot{ }^{15,17}$ Using an EvWaCo focal plane mask, it would be possible to finely 

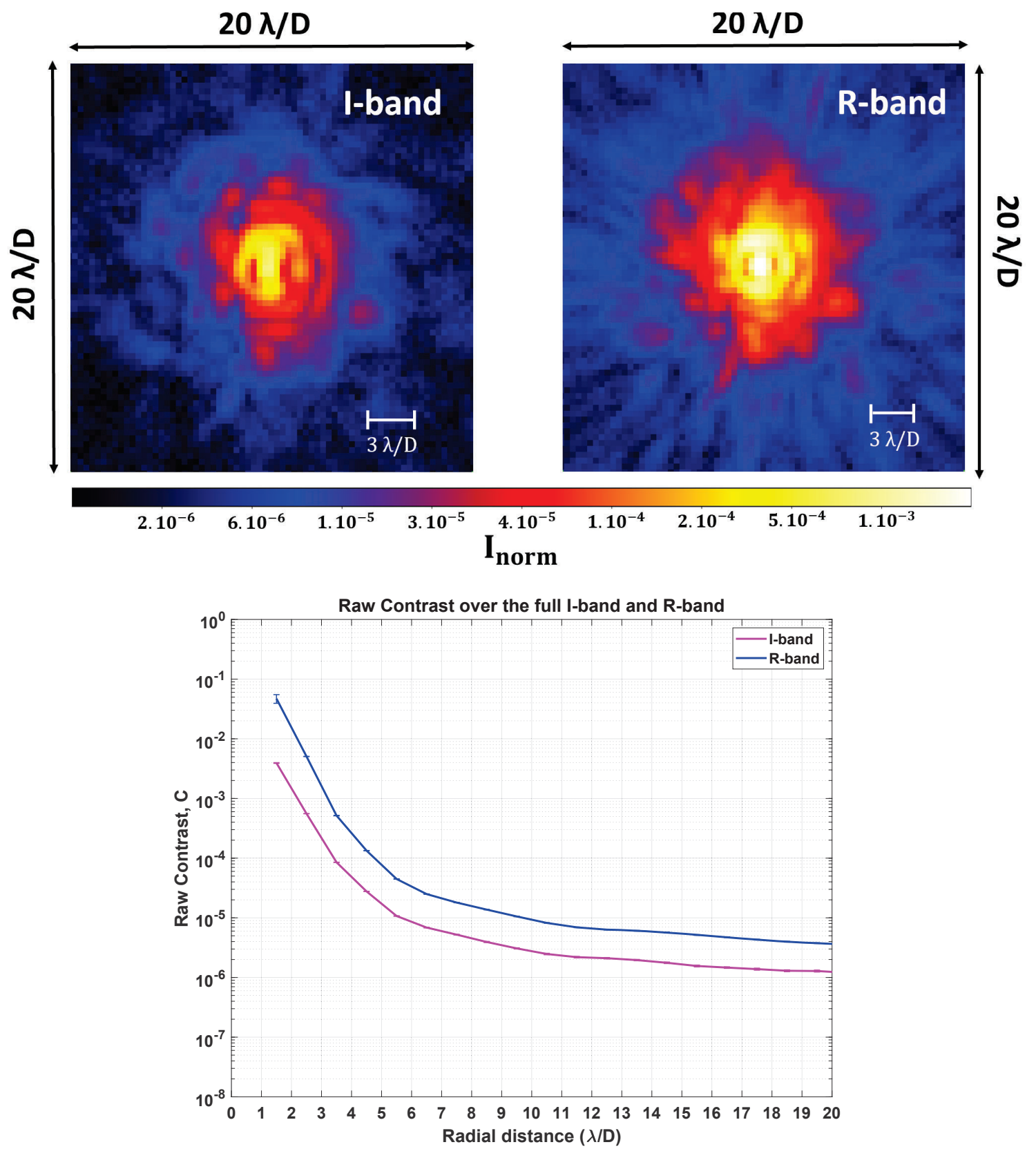

Figure 4. 2D Normalized irradiance distribution over the full I-band (first column) and over the full R-band (second) column and their corresponding raw contrast curves.

tune the size of the mask from $4 \lambda / D(0.27 ")$ to $9.75 \lambda / D(0.65 ")$. Thus, it allows you to study details as close as 0.27 " from the center of the star.

\section{DEVELOPMENT PLAN}

The EvWaCo development plan comprises three distinguished parts: the development of the EvWaCo prototype, the setup upgrade to High Dispersion Coronagraphy and the space qualification of EvWaCo FPM. 


\subsection{EvWaCo prototype}

The EvWaCo prototype is on AIT (Assembly, Integtration, Test) \& AIV (Assembly, Integration, Verification) activities at NARIT Center for Optics and Photonics clean rooms. The first alignment of the coronagraph on the prototype will only include a plane mirror instead of the deformable mirror. We plan to verify the performance of the coronagraph (beam geometry, contrast, IWA) between April and September 2021. During that time period, we will develop and verify the adaptive optics that includes the measurement of the DM influence matrix, the assembly and test of the wavefront sensor, and the development of the Real-Time Control loop.

Between September and December 2021, we plan to install the adaptive optics in the EvWaCo prototype, and optimize the full system while measuring contrasts with AO. Between January and April 2022, we will install the EvWaCo breadboard on the mechanical structure and mount the full prototype vertically on a dummy version of the Thai National Telescope IC. The final optimization and performance verification will be performed between May and August 2022 in the configuration representative of EvWaCo operating conditions at the Thai National Observatory. In September 2022, we plan to install the coronagraph on the TNT and prepare the prototype for the first on-sky tests in October 2022.

Between 2022 and 2025, the prototype and the AO will be optimized during several observation runs with the TNT. The objective will be to perform the first observations with the optimized AO control system. After 2025, we estimate that the EvWaCo prototype will be available for routine observations of the star environment. This instrument will be also be used as a reference tool for students and the astronomers to learn how to operate a high contrast imager (coronagraph equipped with adaptive optics), and to process and analyze the data provided by the system. Our long-term objective is to develop a team of astronomers able to use instruments equipped with $\mathrm{AO}$ and mounted on large and extremely large telescopes and/or space systems.

\subsection{High Dispersion Coronagraph}

The EvWaCo setup has been able to provide deep contrast in both R- and I- bands, thus, confirming the capability to provide deep contrasts over large spectral bands of the proposed concept. One of the most promising applications of this achromatic behavior is the development of a High Dispersion Coronagraph (HDC) by combining the EvWaCo setup with a high resolution spectrograph through single-mode fibers. Using the HDC, raw contrasts close to $10^{-4}$ is already sufficient to detect chemical components of the observed companion. ${ }^{18}$ We plan to combine EvWaCo with the High Resolution Spectrograph EXOhSPEC ${ }^{19}$ already developed at NARIT that is specified to operate at wavelengths [400 nm, $800 \mathrm{~nm}$ ] with a resolution $\mathrm{R} \geq 70,000$. This spectrograph is designed for small core fibers and ready to welcome single mode fibers as an input. It can provide spectrum over R- and I- bands with minor modifications for the grating orientation.

Moving towards a High Dispersion Coronagraph would require upgrading the current testbed such that it will be equipped with an adaptive optics system and will later on be connected to a high resolution spectrograph. This year, we plan to install and optimize the adaptive optics in the testbed. The deformable mirror is from Boston Micromachine with a $12 \times 12$ actuator. By the end of the year, we plan to measure the contrast performance including the detection of a companion in the R- and I-bands using the AO. In parallel, we will be designing and developing apodizers to improve the contrast performance with and without central obscuration and spiders.

Between 2022 and 2024, we will install an injection unit and insert a single mode fiber (SMF) at the EvWaCo image plane to measure the performance in terms of contrast, IWA and throughput at the SMF output. Then, we will connect EvWaCo to EXOhSPEC and perform first companion spectrum measurements. Figure 5 shows the current EXOhSPEC setup at the Thai National Observatory.

Between 2024 and 2025, we will focus our efforts on setup optimization, automation of the measurement process and chemical detection using cross-correlation function. Our aim is to provide routine measurements and open access to space agencies and laboratories for sub-system tests and optimization by horizon 2025.

\subsection{Space qualification}

The Thai Space Consortium, established in 2018, aims at developing the capacities, facilities and technology for space observations and explorations in Thailand. As part of the Thai Space Consortium, the EvWaCo project aims at developing state-of-the-art technologies for high contrast observation with the medium- and long-term 


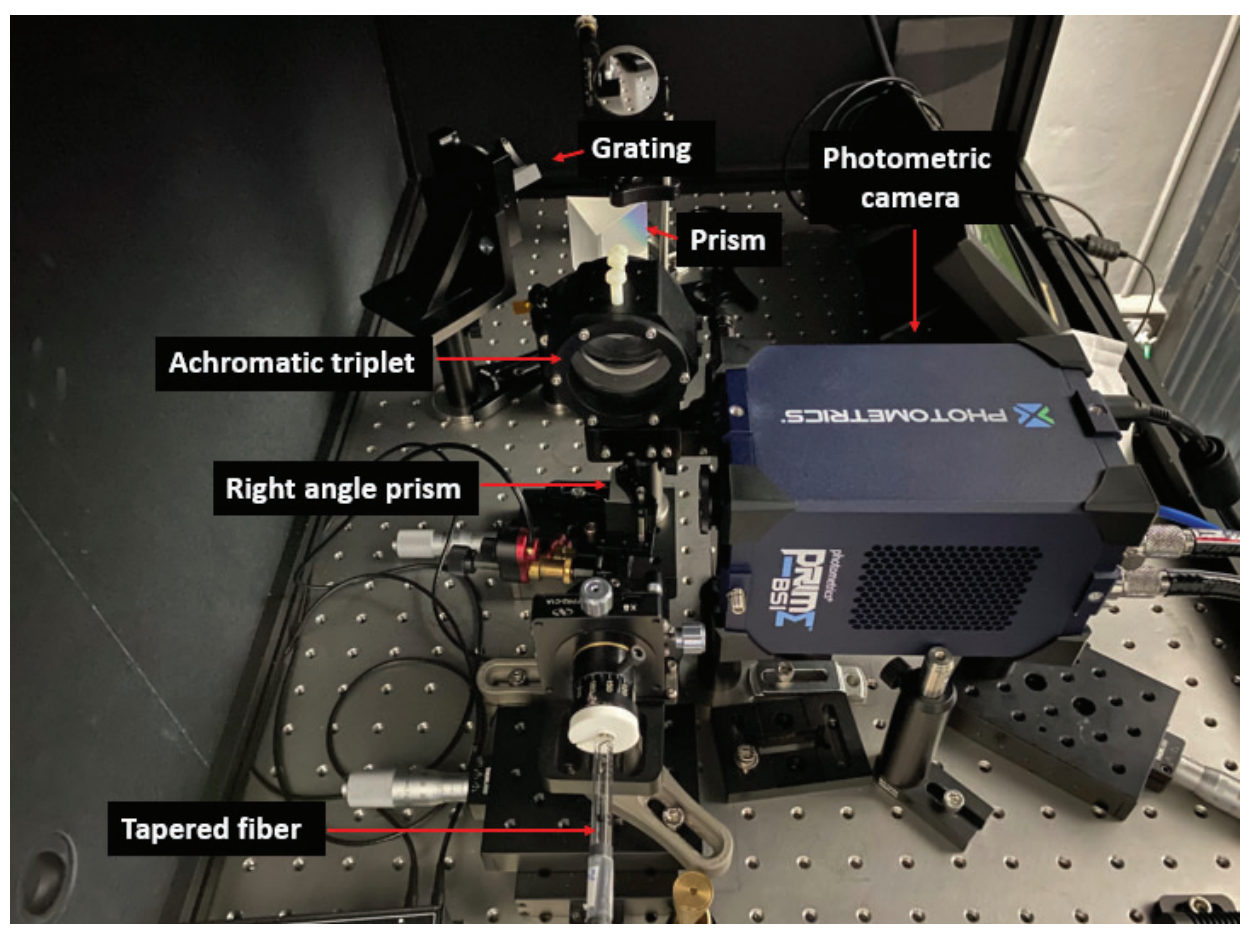

Figure 5. EXOhSPEC setup at the Thai National Observatory

objective of providing sub-systems such as coronagraphic masks, adaptive optics or spectrographs for future space missions to detect and characterize exoplanets.

Under this premise, we will verify that the EvWacO FPM can survive the launch and the space environment. In particular, we will test its response to vibration, shocks, vacuum and thermal cycle.

\section{CONCLUSION}

The EvWaCo project is one of the active fields of research at NARIT Center for Optics and Photonics, and the Thai Space Consortium that aims to install a high contrast imager at the $2.4 \mathrm{~m}$ Thai National Telescope. The breadboard, the materials and the mechanical structure have been carefully selected and designed to meet the strong constraints imposed by both the optical specifications of the instrument and the mechanical tolerance of the Thai National Telescope instrument cube. We have shown that with the current passive setup in the laboratory, we are able to reach a contrast performance of $10^{-4}$ at $3 \lambda / D$ in the I-band and at $4 \lambda / D$ in the Rband. In the future, we hope to improve the performance by designing an apodizer and coupling the coronagraph with a spectrograph to improve the detection and characterization of the companion.

\section{ACKNOWLEDGMENTS}

This work is carried out at the NARIT Center for Optics and Photonics. We thank the mechanical engineers of the Operations Department of NARIT for the fabrication of optical component supports in the testbed.

The Thai Space Consortium (TSC) is an association composed of the National Astronomical Research Institute of Thailand (NARIT), Siam Photon, Geo-Informatics and Space Technology Development Agency (GISTDA), Suranaree University of Technology (SUT), King Mongkut's University of Technology North Bangkok (KMUTNB), King Mongkut's Institute of Technology Ladkrabang (KMITL), and National Innovation Agency (NIA). TSC is funded by PMU-B, Office of National Higher Education Science Research and Innovation Policy 
Council. The research is supported by the Program Management Unit for Human Resources \& Institutional De-

velopment, Research and Innovation, NXPO (Grant number: B05F630115). We also thank the Chulalongkorn University's CUniverse (CUUAASC) grant for the support.

\section{REFERENCES}

[1] Mawet, D., Pueyo, L., Lawson, P., Mugnier, L., and et. al., "Review of small-angle coronagraphic techniques in the wake of ground-based second-generation adaptive optics," in [Space Telescopes and Instrumentation 2012: Optical, Infrared and Millimeter Wave], Clampin, M. C., Fazio, G., MacEwen, H. A., and Jr., J. O., eds., Proc. SPIE 8442 (2012).

[2] Beuzit, J. L., Vigan, A., Mouillet, D., Dohlen, K., Gratton, R., and et. al., "SPHERE: the exoplanet imager for the very large telescope," Astronomy Astrophysics 631 (2019).

[3] Macintosh, B., Graham, J. R., Ingraham, P., Konopacky, Q., Marois, C., and et. al., "First light of the Gemini Planet Imager," PNAS 111, 12661-12666 (2014).

[4] Macintosh, B., Graham, J. R., Barman, T., Rosa, R. D., Konopacky, Q., and et. al., "Discovery and spectroscopy of the young jovian planet 51 Eri b with the Gemini Planet Imager," Science 350, 64-67 (2015).

[5] Wagner, K., Apai, D., Kasper, M., Kratter, K., McClure, M., Robberto, M., and Beuzit, J., "Direct imaging discovery of a jovian exoplanet within a triple-star system," Science 353, 673-678 (2016).

[6] Zhu, S., Yu, A., Hawley, D., and Roy, R., "Frustrated total internal reflection: A demonstration and review," Americal Journal of Physics 54 (1986).

[7] Gross, H., [Handbook of Optical Systems: Volume 1: Fundamentals of Technical Optics], Wiley-VCH Verlag $\mathrm{GmbH}(2005)$.

[8] Rabbia, Y., "Shared constraints and specific characters in very high dynamics imaging," in [EAS Publications Series], Aime, C. and Soummer, R., eds., Proceedings in Astronomy with High Contrast Imaging 8, 65-78 (2003).

[9] Buisset, C., Rabbia, Y., Lepine, T., Alagao, M., Ducrot, E., Poshyachinda, S., and Soonthornthum, B., "Study of a coronagraphic mask using evanescent waves," Optics Express 25, 7273-7287 (2017).

[10] Buisset, C., , Lepine, T., Thiebaut, E., Langlois, M., M.A. Alagao, I. T.-B., Rabbia, Y., Poshyachinda, S., and Soonthornthum, B., "The evanescent wave coronagraph project: setup results and demonstrator preliminary design," in [Advances in Optical and Mechanical Technologies for Telescopes and Instrumentation III], Navarro, R. and Geyl, R., eds., Proc. SPIE 10706 (2018).

[11] Alagao, M. A., Buisset, C., Rabbia, Y., Lepine, T., Poshyachinda, S., and Soonthornthum, B., "Development status and performance of the evanescent wave coronagraph testbed," in [Techniques and Instrumentation for Detection of Exoplanets VIII], Shaklan, S., ed., Proc. SPIE 10400 (2017).

[12] Alagao, M., Kawinkij, A., Buisset, C., Prasit, A., Lepine, T., and et. al., "Deep contrast and companion detection using the evwaco testbed equipped with an achromatic focal plane mask and an adjustable inner working angle," Astronomical Journal (in press, (doi: 10.3847/1538-3881/abe709)).

[13] Ruane, G., Riggs, A., Mazoyer, J., Por, E., M.N'Diaye, E. H., and et. al., "Review of high-contrast imaging systems for current and future ground- and space-based telescopes i. coronagraph design methods and optical performance metrics," in [Space Telescopes and Instrumentation 2018: Optical, Infrared and Millimeter Wave], Lystrup, M., MacEwen, H., Fazio, G., Batalha, N., Siegler, N., and Tong, E., eds., Proc. SPIE 10698 (2018).

[14] Ebbets, D., Sembach, K., and Neff, S., "Cosmic Origins Enabled by the Coronagraph Instrument on NASA's WFIRST/AFTA Mission." NASA Cosmic Origins Program Analysis Group, 4 January 2015 https://cor . gsfc.nasa.gov/copag/aas_jan2015/Ebbets.pdf. (Accessed: 15 February 2021).

[15] Grady, C., Profitt, C., Malumuth, E., Woodgate, B., and et.al., "Coronagraphic imaging with the Hubble Space Telescope and the Space Telescope Imaging Spectrograph," Publications of the Astronomical Society of the Pacific 115 (2003).

[16] Schneider, G., Gaspar, A., Debes, J., Gull, T., and et.al., "Enabling Narrow(est) IWA Coronagraphy with STIS Bar5 and Bar10 Occulters." Instrument Science Report STIS 2017-XX, January 2017 https: //arxiv.org/ftp/arxiv/papers/1708/1708.02643.pdf. (Accessed: 25 February 2021). 
[17] Team, S., "Space Telescope Imaging Spetrograph Instrument Handbook for Cycle 25." Space Telescope Imaging Spetrograph Instrument Handbook for Cycle 25, January 2017 https://www.stsci.edu/files/ live/sites/www/files/home/hst/documentation/_documents/stis/stis_ihb_cycle25.pdf. (Accessed: 15 February 2021).

[18] Wang, J., Mawet, D., Ruane, G., Hu, R., and Benneke, B., "Observing Exoplanets with High Dispersion Coronagraphy. I. The Scientific Potential of Current and Next-Generation Large Ground and Space Telescopes," The Astronomical Journal 153 (2017).

[19] Lhospice, E., Buisset, C., Jones, H., Martin, W. E., Errmann, R., Sithajan, S., Boonsri, C., and et. al., "EXOhSPEC folded design optimization and performance optimization," in [Techniques for Detection of Exoplanet IX], Shaklan, S., ed., Proc. SPIE 11117 (2019). 